\title{
THE IMPLICATIONS OF DISC INSTABILITIES ON CATACLYSMIC VARIABLE STRUCTURE AND EVOLUTION
}

\author{
J.-P. Lasota ${ }^{1}$ \\ RESUMEN
}

Al aplicar el modelo de inestabilidad térmico-viscosa del disco a varias clases de variables cataclísmicas, con frecuencia se requiere que el disco de acreción sea truncado. Arguyo que en la mayoría de los casos este truncamiento interno se debo al campo magnético de la enana blanca.

\section{ABSTRACT}

Applications of the thermal-viscous disc instability model to various classes of cataclysmic variable very often require the accretion disc to be truncated. I argue that in most cases this inner truncation must be due to the white dwarf's magnetic ficld.

\section{Key Words: ACCRETION, ACCRETION DISCS - INSTABILITIES - NOVAE, CATACLYSMIC VARIABLES - STARS: EVOLUTION}

\section{THE MAGNETIC NATURE OF VY SCL STARS}

VY Scl stars are very bright, Cataclysmic Variables (CVs) which occasionally undergo a diminution in brightness of more than one magnitude. Such drops in luminosity should bring the accretion discs in these systems into the dwarf nova instability strip, but surprisingly 110 outbursts are observed. As pointed out by Lasota, Hameury \& Huré (1995) outbursts would be prevented if the disc were truncated at radius

$$
r_{\mathrm{tr}}>r_{\mathrm{crit}} \approx 6 \times 10^{9}\left(\frac{\dot{M I}}{10^{15} \mathrm{~g} \mathrm{~s}-1}\right)^{0.375} M_{1}^{0.333} \mathrm{~cm}
$$

where $r_{\mathrm{tr}}$ is the transition radius and $M_{1}$ is the whitedwarf mass in solar units (sec c.g. Lasota 2001). Such truncation removes the imner, unstable parts of the disc. This stabilization can be achieved either by the action of the white dwarf's magnetic field or by heating and/or evaporation of the unstable inner regions. Leach et al. (1999; sce also King 1997; Hameury, Lasota \& Dubus 1999) proposed that heating by the accretion-heated white dwarf in highly accreting VY Scl stars brings their inner disc into a hot and stable state. In such a case the transition radius would be (Hameury \& Lasota 2002, hereafter HL02):

$$
r_{\mathrm{tr}} \approx 6.7 T_{* .40}^{4 / 3}\left(\frac{T_{\text {crit }}}{6500 \mathrm{~K}}\right)^{-4 / 3} R_{*},
$$

\footnotetext{
${ }^{1}$ Institut d'Astrophysique de Paris, France
}

where it is assumed for simplicity's sake that a disc is thermally stable if its photospheric temperature is greater than $T_{\text {crit }} \simeq 6500 \mathrm{~K}, T_{*, 40}=T_{*} / 40,000 \mathrm{~K}$ and the albedo $=0$ (which obviously maximizes the transition radius). Therefore a $0.7 \mathrm{M}_{\odot}$ white dwarf would stabilize the disc only if heated to more than $50,000 \mathrm{~K}$ and more massive stars would have to be heated to temperatures that have never been observed. In any case, RU Peg, the CV with the hottest $(50,000 \mathrm{~K})$ white dwarf observed (Sion \& Urban 2002), is a dwarf nova, so at least in this case white-dwarf heating does not work.

In spite of these arguments and observations Hoard et al. (2003) assert that the VY Scl star DW UN la could be stabilized by white dwarf heating because it has the right temperature $(50,000 \mathrm{~K})$ and its radius, due to heating, could be much larger than one would deduce from its mass $\left(0.8 \mathrm{M}_{\odot}\right)$. Unfortunately the argument cited in favour of oversized white dwarfs (Koester \& Schoenberner 1986) does not apply to the old primaries in CVs. Fortunately, however, the case of DW UMa provides a very strong argument in favor of a truncation that cannot be achieved by any existing hot white dwarf. As argued by HLO2 the real problem is not so much with explaining the absence of outbursts during low states as explaining their absence during long intermediate states. During a very long transition from a low to a high state DW UMa showed no outbursts. The 4 mag rise lasted $\sim 500$ days (Honeycutt et al. 1993), therefore the disc would have crossed the instability strip in a quasi-stationary way, because its viscous time is only $\sim 10-20$ days. This is 


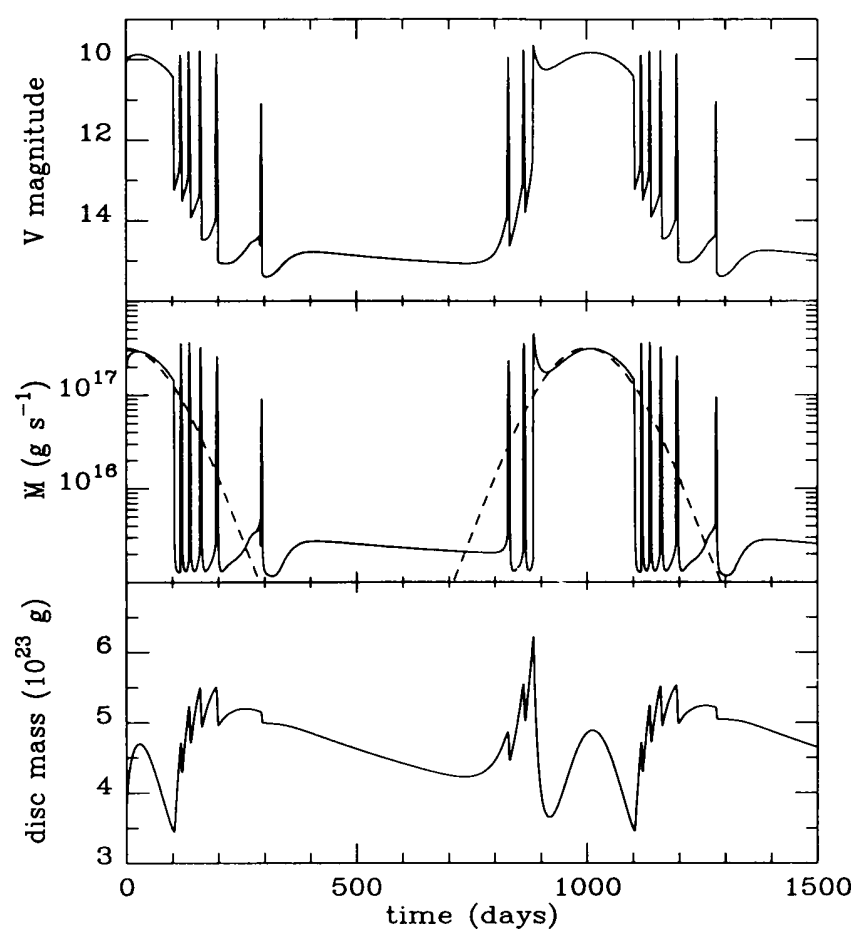

Fig. 1. Top panel : visual light curve of a binary system in which the mass-transfer rate slowly varies. The accretion disc is disrupted by the magnetic field of a $0.7 \mathrm{M}$ white dwarf with $\mu_{30}=10$. Intermediate panel : mass accretion rate onto the white dwarf (solid line), and mass transfer rate (dashed line). Bottom panel : disc mass

illustrated by Fig. 1 from HL02, which shows the light-curve of a system in which the disc truncation prevents outbursts during quiescence, while a frantic outburst activity is observed during intermediate states. The only thing that would prevent outbursts during long intermediate states would be the absence of the disc, i.e. the transition radius should be at least of the order of the circularization radius, say. Therefore despite Hellier's (this volume) comment that “... Hameury \& Lasota (2002) prefer a model in which a strong magnetic field evacuates the inner disc", the question is not of preference, strong field or inner disc. In order for the inner disc to be evacuated there must be typically magnetic moments $\mu=5 \times 10^{30} \mathrm{G} \mathrm{cm}^{3}$, therefore rather weakish magnetic fields. But the absence of outbursts during intermediate states requires the evacuation of the "whole" disc, and this does indeed require stronger fields: $\mu \gtrsim 4-7.5 \times 10^{32} \mathrm{G} \mathrm{cm}^{3}$, i.e. magnetic moments expected in some Intermediate Polars (IP).

This would imply that variable circular polarization should be detected, at least in such VY Scl stars as DW UMa or MV Lyr in which long intermediate states are observed. One should keep in mind, however, that circular polarization has been detected only in five IPs out of a total of more than 30 - those that harbor white dwarfs with the highest magnetic fields $\sim 2-8 \mathrm{MG}$, the highest value corresponding to V2400 Oph (Buckley et al. 1995). Since VY Scl stars would have rather lower fields, the detection of their circular polarization could be rather difficult.

Another signature of the presence of a magnetized white dwarf takes the form of periodic oscillations and none seem to be detected so far in $V Y$ Scl stars. But such detection could be difficult. as shown by the case of one of the best-observerd cataclysmic variables, the SU UMIa type dwarf nova OY Car. Indications of the presenc' of a magnetic: white dwarf in this short period $\left(P_{\text {orb }}=1.51 \mathrm{~h}\right)$. eclipsing $\left(i=83^{\circ}\right)$ and not very distant $(\sim 82 \mathrm{pc})$ have been found only recently (Ramsay et al. 2001a; Hakala id Ramsay 2003; Wheatley \& West 2003). The evidence comes from X-ray observations of $O Y$ ( $\mathrm{Ar}$ in quiescence. Considering that it took more than twenty years to find hints of the magnetic nature of this so frequently observed system and that the evidence is still not conclusive it should not be surprising that observations of VY Scl stars have not yet provided such evidence. The nature of their Xray emission is subject to controversy which could be resolved by Chandra and XMM grating spectra observations (Mauche \& Mukai 2002).

\section{MAGNETIC DWARF NOJAE}

If magnetic, OY Car would have a truncated disc in quiescence. It is too often forgotten that in quiescence dwarf-nova accretion disc's are unsteady, i.e. the accretion rate through the disc is not constant. According to the Disc Instability Model (DIN) the quiescent accretion rate in the disc $\dot{M}_{\mathrm{G}}(r)$ must be everywhere smaller than the critical rate $\dot{M}_{\mathrm{cr}}$ :

$$
\dot{M}_{\mathrm{q}}<\dot{M}_{\mathrm{cr}}=9 \times 10^{12}\left(\frac{M_{1}}{M_{y}}\right)^{-0 . \times 8} r_{!)}^{2.65} \mathrm{~g} \mathrm{~s}^{-1} \text {. }
$$

where $r_{9}$ is the radius in units of $10^{9} \mathrm{~cm}$. Therefore in a quiescent disc extending down to the surface of the accreting star the inner accretion rate would be very low, in general more than two orders of magnitude lower than the rate at which matter is transferred from the companion to the outer disc regions. But $\mathrm{X}$-ray observations of quiescent dwarf. nowar often imply accretion rates higher than the critical rate near the white dwarf's surface. The accretion rate producing the observed X-ray luminosity $L_{X}$ can be estimated as

$M_{\mathrm{X}}=7.5 \times 10^{13} \eta_{X}^{-1} r_{9}\left(\frac{M_{1}}{M_{\odot}}\right)^{-1}\left(\frac{L_{X}}{10^{31} \mathrm{erg} / s}\right) \mathrm{g} s^{-1}$. 
where $\eta_{\mathrm{X}}$ is the efficionc $\mathrm{y}$ of producing $\mathrm{X}$-rays. From the estimated bolometric X-ray luminosity $L_{X} \sim$ $4 \times 10^{30} \mathrm{erg} \mathrm{s}^{-1}$ of OY Car in quiescence. Ramsey et al. (2001b) one can deduce, for a $1 M_{c,}$, white dwarf. an accretion rate $\dot{M}_{\mathrm{X}} \approx 1.5 \times 10^{13} \eta_{X}^{-1} \mathrm{~g} \mathrm{~s}^{-1}$, whereas the critical rate near the white dwarf's surface is $\sim 1.4 \times 10^{12} \mathrm{~g} \rightarrow$ ', i.e. at least $\left(\eta_{x}<1\right)$ ten times lower. Taken at fare value this would mean that the inner disc in OY Car when it is quiescent is unstable. Clearly it is not. As pointed out by Meyer \& Meycr-Hofneister (1994) a truncated disc would remove this inconsistency. (These authors argne in favour of disc muncation by evaporation.) This would require the immer clime radius to be

$$
r_{\mathrm{in}}>9.6 \times 10^{9} \eta_{X}^{-0.61} M_{1}^{0.6 \mathrm{i \tau}}\left(\frac{L_{X}}{10^{31} \mathrm{erg} / \mathrm{s}}\right)^{0.61} \mathrm{~cm}
$$

which for OY Car would recuire $\mu \gtrsim 8 \times 10^{30} \mathrm{G} \mathrm{cm}^{3}$.

This is slightly higher than the magnetic moment $\left(2 \times 10^{30} \mathrm{G}\left(\mathrm{m}^{3}\right)\right.$ producing the truncation required to reproduce the olserverl quiescent X-ray luminosity and the time-lags observed in the X-ray, EUV, UV and optical emission of SS Cyg (Schreiber et al., 2003).

\section{WZ SGE AS A MAGNETIC ROTATOR}

The WZ Sge 27.87 s coherent oscillation was first attributed to the rotation of all accreting magnetized white dwarf by Patterson (1980). This oscillation disappeared during the 1978 outburst and was absent for about $16 \mathrm{y}$ ars (although a $28.96 \mathrm{~s}$ oscillation reappeared when $\mathrm{WZZ}$ Sge was at twice its pre-outburst brightness). Although it wats easy to see that during the outburst the increased accretion rate could suppress the magnetosphere the lack of the principal pulse afterwards was harder to understand and cast a shadow of doubt on the presence of a rapidly rotating white dwarf in $1 \mathrm{IZ} /$ Sge. However. in 1995 the $28 \mathrm{~s}$ oscillation reappeared and was detected also in the $2-6 \mathrm{keV}$ ASC'A encrgy band (Patterson of al. 1998). This strengthened the case for the presence of a rotating, magnetized white dwarf in WZ Sge, especially since UV spertral observations (Cheng ot al. 1997) confinmed the presence of a rapidly rotating white dwarf. Then, $11 \%$ Sge unexpertedly $(\sim 7$ vears carly) wont to outburst in 2001 and once again the $28 \mathrm{~s}$ signal was lost. Observat ions during the decline and after the system settled back to quicsence led to 1 wo rather confusing conclusions. First, the 29 s oscillation. which reappeared early as rluring the 1978 outburst. Was shown to be independent of the white dwarf's temperature (Wolsh et al. 2003), thus ruling out the the white dwarf nonradial g-mode pulsation hypothesis which had proposed as an alternative to positing a rotational origin to the oscillations. Interestingly, such pulsations have been recently detected in GW Lib (ran Zyl ct al. 2004) a "near twin of WZ Sge" (Thorstensen et al. 2002). On the other hand the 27.87s is again absent, this time also in X.MN-Newton data which obviously challenges the magnetic rotator model of WZ Sge (Mukai, these proceedings). Of course one should remember that after the 1978 outburst there was a wait of 16 years before this signal reappeared. So maybe it was risky for the present author to bet a bottle of "Paradigm" wine that the $28 \mathrm{~s}$ would reappear after only 7 years after the last outburst. The hope lies in the high sensitivity of XMM-Newton. In any case one has to agree with Koji Mukai that "if it [WZ Sge] is magnetic it does not resemble any other magnetic binary we know about. This in fact was the verdict of Lasota. Kuulkers \& Charles (1999), who concluded that WZ Sge is in an 'ejector' phase, ejecting most of the transferred matter, in a state "totally different from the usual IPs". WZ Sge is most probably in a state intermediate between that of $\mathrm{AE} \mathrm{Aqr}$ - a pure discless ejector (Wynn et al., 1997) and that of DQ Her which seems to have a steady accretion disc. No model of such a flow has been yet published but one should appear soon (Wynn, private communication). If the white dwarf in WZ Sge is magnetic we have to try to understand how it has been spun up to its present rate. The only way to achieve this is through accretion disc spin-up. The white dwarf spin rate increases as it accretes the Keplerian angular momentum of matter at the inner disc edge. An equilibrium period is reached when angular momentum is accreted at the same rate as it is centrifugally expelled by the spinning white dwarf. In the case of a magnetized white dwarf the equilibrium period is equal to the Keplerian period of the magnetosphere $\left(\omega_{s}=1\right)$. The equilibrium spin would be then

$$
P_{\text {eq }}=360 M_{1}^{-5 / 7} \dot{\Lambda}_{15}^{-3 / 7} \mu_{31}^{6 / 7} \mathrm{~s},
$$

From Eq. (5) and the quiescent X-ray luminosity $L_{X} \sim 3 \times 10^{30} \mathrm{erg} \mathrm{s}^{-1}$ one can deduce a magnetic moment $\sim 10^{31} \mathrm{G} \mathrm{cm}^{3}$. According to the ejector model of Lasota, Kuulkers \& Lasota (1999) the magnetic moment could be even greater. This means that an accretion rate $\gtrsim 10^{17} \mathrm{~g} \mathrm{~s}^{-1}$ was required to bring $\mathrm{IZZ}$ Sge to its present very rapid rotation rate. Such aceretion rates are typical of nova-like CVs at orbital periods longer than 3 hours whereas WZ Sge is very close to the ninimum period for CVs where secular mass-transfer rates are two orders of magnitude 
lower. The present mass transfer rate in WZ Sge is in good agreement with that predicted by mass loss from the secondary driven by gravitational radiation alone. However, fluctuations of the mass-transfer rate on time-scales of the order of the white dwarf spin-up time, i.e. $10^{4}-10^{5}$ years, would not modify the secular evolution of the binary. The hypothesis of high mass-transfer rate episodes in the life of WZ Sge is supported by the existence of ER UMa stars. These systems have orbital periods between 79 and 92 minutes and should have high $\left(\sim 10^{17} \mathrm{~g} \mathrm{~s}^{-1}\right)$ mass transfer rates, which would explain their extremely short intervals between superoutbursts (19-44d) and very short $(3-4 d)$ 'normal' outburst intervals. They are the high accretion-rate equivalent of the low accretion rate systems SU UMa's (OY Car is such a system, WZ Sge is a SU UMa system showing superoutbursts only). The idea that DI UMa (one of the 4 known ER UMa's with $P_{\text {orb }}=79 \mathrm{~min}$ ), which is more luminous than WZ Sge by a factor $\sim 50$, spends most of its life as 'an ordinary WZ Sge star', but was caught during an 'upward surge in accretion', has been proposed by Patterson (1998). The white dwarf in WZ Sge was spinning at the equilibrium rate when this system was an ER UMa star. After some time the mass transfer rate would return to its secular value, two orders of magnitude lower than that required for $P_{\text {eq }} \sim$ few $\times 10$ s. In a very short time (the viscous time of the disc $\sim$ days) the magnetosphere will start expanding and becoming larger than the corotation radius. The system enters into the ejector phase. It will stay in this, WZ Sge-phase (about $10^{5}$ years) until it gets to a new spin equilibrium corresponding to the low accretion rate ( $\sim$ a few minutes). HT Cam is a CV with exactly such properties: $P_{\text {orb }}=86 \mathrm{~min}, P_{\text {spin }}=8.6$ min. The spin period corresponds to the equilibrium value as given by Eq. (6) for the expected secular mass-transfer rate. One can therefore expect the white dwarf's magnetic field in HT Cam to have a strength close to that of WZ Sge. and this system would be a WZ Sge-type system at a particular phase of its spin-up/spin-down history. A jump in the mass transfer rate could bring such an equilibrium system back to a spin-up phase. If HT Cam does indeed have a magnetic field close to that of WZ Sge and if its mass-transfer rate is close the secular rate, its accretion disc should be truncated at a radius close to the value given by Eq. (1) and therefore stable with respect to the thermal-viscous instability. HT Cam shows very brief outbursts (Ishioka et al. 2002; Kemp et al. 2002) that could reflect the marginal stability of its disc.

\section{CONCLUSION}

There is growing evidence that accretion discs in CVs are truncated. Although I prefer to posit a magnetic origin for this effect. I cannot preclude that in some cases it is due to disc evaporation as proposed by Meyer \& Meyer-Hofmeister (1994), especially since truncation is also required in accretion onto black holes (e.g. Dubus, Hameury \& Lasota 2001) where the magnetic field cannot do the job.

\section{REFERENCES}

Buckley, D. A. H., Sekiguchi, K., Motch, C. et al. 1995. MNRAS, 275, 1028

Cheng, F. H., Sion, E. M., Szkody, P., \& Huang, M. 1997. ApJL, 484, L149

Dubus, G., Hameury, J.-MI., \& Lasota, J.-P. 2001. A\&-A. 373,251

Honeycutt, R.K., Robertson, J.W..199ฬ. A.J, 116, 1961

Hakala, P., Ramsay, G. 2003, Ad-1. submitted, astroph/0312343

Hameury, J.-M. \& Lasota, J.-P. 2002, A\&.A. 394, 2:31

Hameury, J.-M., Lasota, J.-P.. \& Dubus, G. 1999. MNRAS, 303, 39

Hoard, D. W., Szkody, P., Froning, C. S., Long, K. S.. \& Knigge, C. 2003, AJ, 126, 2473

Ishioka, R. et al. 2002, PASJ, 54, 581

Kemp, J., Patterson, J., Thorstensen, J. R. et al. 2002. PASP, 114, 623

King, A.R. 1997, MNRAS, 288, L16

King, A.R., \& Lasota, J.-P. 1991, ApJ, 378, 674

Koester, D. \& Schoenberner, D. 1986, A\&A, 154. 125

Lasota, J.-P. 2001, NewAR, 45, 449

Lasota, J.-P., Hameury, J.-M., \& Huré, J.-M. 1995, ApJ. 302, L29

Lasota, J.-P., Kuulkers, E., \& Charles, P. 1999, MNRAS. 305,473

Leach, R., Hessman, F.V., King, A.R., Stehle. R.. \& Mattei, J. 1999, MNRAS, 305, 225

Mauche, C. W. \& Mukai, K. 2002, ApJL. 566. L33

Meyer, F. \& Meyer-Hofmeister, E. 199-1. AdA. 288. 175

Patterson, J. 1980, ApJ, 2.11, 235

Ramsay, G. et al. 2001a, A\&A, 365, L288

Ramsay, G. et al. 2001b, A\&A, 365, L294

Schreiber, M. R., Hameury, J.-M., \& Lasota, J.-P. 2003. A\&A, 410, 239

Sion, E. M. \& Urban, J. 2002, ApJ, 572. 456

Thorstensen, J. R, Patterson, J., Kemp, J.. d lennes. S. 2002, PASP, 114. 1108

Tovmassian, G.H., et al. 1998. A\&A. 335. 2.27

van Zyl, L. et a!., 2004, MNRAS, in press, astroph/0401459

Welsh, W. F., Sion, E. M., Godon, P. 2003, ApJ, 504, 509

Who'atley, P. J. \& Wost. R. G. 2003, MNRAS. 345. 1009

Wynn, G. A., King, A. R.. \& Horne, K. 1997. MNR.As. 286,436 